\title{
Screening Analysis of 10 Adrenal Steroids by Matrix-Assisted Laser Desorption Ionization-Tandem Mass Spectrometry
}

\author{
Sun Ju Kim ${ }^{\text {a,b }}$, Hyun-Jin Jung ${ }^{\text {a }}$, Bong Chul Chung ${ }^{\text {a }}$, and Man Ho Choi ${ }^{\text {a,b,* }}$ \\ ${ }^{a}$ Future Convergence Research Division, Korea Institute of Science and Technology, Seoul 136-791, Korea \\ ${ }^{b}$ Department of Biomolecular Science, University of Science and Technology, Daejeon 305-333, Korea
}

Received September 3, 2011; Revised September 13, 2011; Accepted September 13, 2011

First published on the web September 15, 2011; http://dx.doi.org/10.5478/MSL.2011.2.3.069

\begin{abstract}
Defective synthesis of the steroid hormones by the adrenal cortex has profound effects on human development and homeostasis. Due to the time-consuming chromatography procedure combined with mass spectrometry, the matrix-assisted laser desorption ionization method coupled to the linear ion-trap tandem mass spectrometry (MALDI-LTQ-MS/MS) was developed for quantitative analysis of 10 adrenal steroids in human serum. Although MALDI-MS can be introduced for its applicability as a high-throughput screening method, it has a limitation on reproducibility within and between samples, which renders poor reproducibility for quantification. For quantitative MALDI-MS/MS analysis, the stable-isotope labeled internal standards were used and the conditions of crystallization were tested. The precision and accuracy were $3.1 \sim 35.5 \%$ and $83.8 \sim 138.5 \%$, respectively, when a mixture of $10 \mathrm{mg} / \mathrm{mL} \alpha$-cyano-4-hydroxycinnamic acid in $0.2 \%$ TFA of $70 \%$ acetonitrile was used as the MALDI matrix. The limit of quantification ranged from 5 to $340 \mathrm{ng} / \mathrm{mL}$, and the linearity as a correlation coefficient was higher than 0.988 for all analytes in the calibration range. Clinical applications include quantitative analyses of patients with congenital adrenal hyperplasia. The devised MALDI-MS/MS technique could be successfully applied to diagnosis of clinical samples.
\end{abstract}

Key words: Adrenal steroid, Congenital adrenal hyperplasia, MALDI-MS, Newborn screening

\section{Introduction}

The steroid hormones secreted by the adrenal cortex are synthesized from cholesterol by a sequence of enzymecatalyzed reactions; this is important for the understanding of metabolic diseases. The levels of adrenal steroids and the activities of their metabolic enzymes have been commonly evaluated by mass spectrometry combined with chromatography. The gas chromatography-mass spectrometry (GC-MS) method showed good sensitivity but is time consuming and may be affecting thermal decomposition during both chemical derivatization and high temperature analysis. ${ }^{1-3}$ As an alternative method, liquid chromatography-mass spectrometry (LC-MS) can also provide a good level of sensitivity but it has been hampered from long separation time and ion suppression, where metabolites are poorly ionized when in the presence of charged ions. ${ }^{4}$

In contrast, the matrix-assisted laser desorption ionization coupled to MS (MALDI-MS) can achieve a high sample throughput, making it attractive to analytical works for increasing productivity and efficiency ${ }^{5-7}$ However, the organic matrices in MALDI analysis are likely to result in coincidental background noise at mass in the low $\mathrm{m} / \mathrm{z}$ range, and these ions are likely in higher abundance then the analyte ions.

*Reprint requests to Dr. Man Ho Choi

E-mail:mh_choi@kist.re.kr
For this reason, many techniques have been addressed: 1) Use of higher molecular weight matrices (such as porphyrins) is an approach that depends on the high molecular weight of the matrix and the small molecule to be effectively analyzed. ${ }^{8}$ Due to a very large proton affinity of porphyrins, many analytes, including steroids, cannot be charged by protonation. 2) Alkali metal ions are preferred in MALDI analysis of molecules lacking basic sites. ${ }^{9}$ ) A binary matrix mixture with different proton affinity has been used to reduce background noise and in consequence improve detection sensitivity. In this manner, the conventional matrix such as CHCA ( $\alpha$-cyano4-hydroxycinnamic acid) owns an acidic group site which makes it ideal for analyte protonation, whereas the amine group of 9-AA (9-aminoacridine) is easily protonated and helps to produce negatively charged species. ${ }^{10,11}$

To quantify compounds with molecular weights $<500 \mathrm{Da}$, structure-specific precursor /product ion combinations in tandem mass spectrometry (MS/MS) have been effectively introduced. ${ }^{2,6}$ Here, the conventional MALDI matrix in lipid analysis, CHCA, was conducted and optimized with linear-ion trap quadrupole (LTQ) MS/MS for effective quantitative profiling of serum adrenal steroids. This method was applied to quantify $17 \alpha$-hydroxy-progesterone (17 $\alpha-\mathrm{OHP})$ in serum samples of congenital adrenal hyperplasia $(\mathrm{CAH})$ patients, because $17 \alpha-\mathrm{OHP}$ is known to be a marker steroid for the diagnosis of 21-hydroxylase deficiency in $\mathrm{CAH}^{12,13}$ 


\section{Experimental}

\section{Chemicals}

All reference standards used in this study are listed in Table 1. Ten endogenous adrenal steroids and three internal standards (IS) were obtained from Steraloids (Newport, RI) and Cambridge Isotope Laboratories (Andover, MA). The $\alpha$-cyano-4-hydroxycinnamic acid as the MALDI matrix was purchased from Sigma Co. (St. Louis, MO). Oasis HLB ${ }^{\mathrm{TM}}$ (3 mL, $60 \mathrm{mg}$; Waters, Milford, MA), which was preconditioned with $3 \mathrm{~mL}$ of methanol followed by $3 \mathrm{~mL}$ of deionized water, was used for solid-phase extraction (SPE). Sodium acetate (reagent grade) and acetic acid (glacial, 99.99+\%) were purchased from Sigma.

All organic solvents used were either analytical or HPLC grade and were purchased from Burdick \& Jackson (Muskegan, MI). Deionized water was prepared using a Milli-Q purification system (Millipore; Billerica, MA).

\section{Preparation of standard solutions}

Stock solutions of all reference standards, including internal standards, were prepared at a concentration of $1 \mathrm{mg} / \mathrm{mL}$ in methanol. Each working solution was diluted with methanol at varied concentrations in the range of 0.1 to $100 \mu \mathrm{g} / \mathrm{mL}$. All standard solutions were stored at $20^{\circ} \mathrm{C}$ until required.

\section{Sample preparation}

The serum samples $(0.4 \mathrm{~mL})$ were added to $2.6 \mathrm{~mL}$ of acetate buffer ( $\mathrm{pH} 5.2)$ and $15 \mu \mathrm{L}$ of three ISs $(5 \mu \mathrm{g} / \mathrm{mL}$ of $d_{9}$-progesterone, $d_{8}$-17 $\alpha$-hydroxyprogesterone and $d_{4}$-cortisol). The samples were extracted with Oasis HLB SPE cartridges placed in a device fitted with a small peristaltic pump and operated at a low flow rate $(<1 \mathrm{~mL} / \mathrm{min})$. After loading the sample onto the cartridge, it was washed with $2 \mathrm{~mL}$ water and eluted twice with $2 \mathrm{~mL}$ of methanol. The eluate obtained was evaporated to dryness under a stream of nitrogen and then $1 \mathrm{~mL}$ of $0.2 \mathrm{M}$ acetate buffer (pH 5.2) was added. The solution was extracted twice with $2.5 \mathrm{~mL}$ of $n$-hexane: ethylacetate $(3: 2, \mathrm{v} / \mathrm{v})$ by mechanical shaking for $10 \mathrm{~min}$. The solution was centrifuged at $2500 \mathrm{rpm}$ for $5 \mathrm{~min}$ and frozen at $-20^{\circ} \mathrm{C}$ to separate the organic layer from the aqueous layer. The separated organic layer was evaporated using a $\mathrm{N}_{2}$ evaporator at $40^{\circ} \mathrm{C}$ and further dried in a vacuum desiccator over $\mathrm{P}_{2} \mathrm{O}_{5} / \mathrm{KOH}$ for at least $30 \mathrm{~min}$. The dried residue was reconstituted with $10 \mu \mathrm{L}$ of $0.2 \%$ trifluoroacetic acid in $70 \%$ acetonitrile and was mixed with an equal volume of the matrix solution $(10 \mathrm{mg} / \mathrm{mL}$ CHCA in $0.2 \%$ trifluoroacetic acid of $70 \%$ acetonitrile). The sample mixture was centrifuged at $1000 \mathrm{rpm}$ for $30 \mathrm{~s}$ in a centrifugal filter (Durapore PVDF, $10 \mathrm{um}$; Millipore, Billerica, MA) and $1 \mu \mathrm{L}$ of the mixture was spotted onto a stainless steel MALDI-target. The resulting sample on the target was subjected to the MALDI-MS in the selected reaction-monitoring (SRM) mode.

\section{Analytical conditions}

A Finnigan LTQ linear ion trap coupled to the Finnigan MALDI (Thermo Finnigan, San Jose, CA) ion source was used for the MALDI-MS/MS analysis. The data were acquired by the Xcalibur 2.0.7 software in the data dependent mode. The pulsed nitrogen was emitted at $337 \mathrm{~nm}$ and the extraction voltage was $20 \mathrm{kV}$. A survey mass scan was followed by MS/MS scans on the most abundant ions for selection of a SRM transition. Each sample spot was repeated three times.

\section{Method validation}

To test the linearity, the calibration standards at nine different concentrations, ranged from 0.1 to $3 \mu \mathrm{g} / \mathrm{mL}$, were prepared by diluting the working solution. Each calibration was then

Table 1. The MALDI-MS/MS information for adrenal steroids analyzed in human serum samples.

\begin{tabular}{|c|c|c|c|c|c|c|c|}
\hline Compound & MW & Precursor ion & Product ion & NCE $(\%)$ & Act Q & Act Times & Isolation Width (IW) \\
\hline Progesterone (Prog) & 314.46 & 315.4 & $\underline{97}, 215,279$ & 60 & 35 & 0.250 & 0.5 \\
\hline $17 \alpha-\mathrm{OH}-\mathrm{Prog}$ & 330.46 & 331.4 & $\underline{253}, 271,295$ & 70 & 35 & 0.250 & 0.5 \\
\hline $11 \alpha-\mathrm{OH}-\mathrm{Prog}$ & 330.46 & 331.4 & $\underline{253}, 271,295$ & 68 & 35 & 0.250 & 0.5 \\
\hline Cortisol (F) & 362.46 & 363.3 & $309,327,327$ & 75 & 35 & 0.250 & 0.5 \\
\hline Cortisone (E) & 360.44 & 361.3 & $\underline{163}, 325,325$ & 68 & 35 & 0.250 & 0.5 \\
\hline Corticosterone (B) & 346.46 & 347.4 & $\underline{265}, 283,311$ & 70 & 35 & 0.250 & 0.5 \\
\hline 11-DehydroB & 344.44 & 345.4 & $269,121,309$ & 70 & 35 & 0.250 & 0.5 \\
\hline 11-DeoxyB & 330.46 & 331.4 & $\underline{277}, 109,295$ & 72 & 35 & 0.250 & 0.5 \\
\hline 11-DeoxyF & 346.46 & 347.4 & $\underline{269}, 299,311$ & 72 & 35 & 0.250 & 0.5 \\
\hline Aldosterone & 360.44 & 361.4 & $\underline{255}, 325$ & 65 & 35 & 0.250 & 0.5 \\
\hline Prog- $d_{9}$ & 323.52 & 324.4 & $\underline{305}, 100,288$ & 65 & 35 & 0.250 & 0.5 \\
\hline $17 \alpha-\mathrm{OH}-$ Prog- $d_{8}$ & 338.51 & 339.5 & $\underline{277}, 258,303$ & 75 & 35 & 0.250 & 0.5 \\
\hline Cortisol- $d_{4}$ & 366.48 & 367.3 & $331,313,311$ & 72 & 35 & 0.250 & 0.5 \\
\hline
\end{tabular}

Quantitative ions are underlined. 
subjected to the sample preparation steps described above. Least-squares regression analysis was performed with the intensity ratio of the analyte over the internal standard against increasing amounts to plot calibration curves.

The limit of detection (LOD) of each steroid was estimated based on the lowest concentration giving by blank matrix standard deviation over the slope of calibration. Each limit of quantification (LOQ) was calculated based on the 3.3 times LOD calculated concentration. The precision was determined by assaying triplicates of the serum samples at each of three different concentrations (low, medium, high) and was expressed as the coefficient of variation $(\% \mathrm{CV})$. For within-day repeatability, five replicates were analyzed. Recovery of each steroid was assessed by comparing the response serum spiked before sample preparation. The recovery was expressed as a percentage of the response of a control sample to which steroids had been added at the same nominal concentration. The recovery of three ISs used was determined individually at the point where concentration spiked, which was used in this study. The storage stability of steroids as their respective derivatives was tested at concentrations range of 1.0 and $3.0 \mu \mathrm{g} / \mathrm{mL}$, and then was compared against freshly prepared $100 \%$ controls analyzed under identical conditions.

\section{Results and Discussion}

\section{Optimization of MALDI matrix for serum adrenal steroids}

In MALDI-MS analysis of adrenal steroids, the types of acidic solvent for protonation, amounts of dissolving solvent and concentration of $\alpha$-cyano-4-hydroxycinnamic acid (CHCA) were tested (Supplementary Figure 1). In addition,

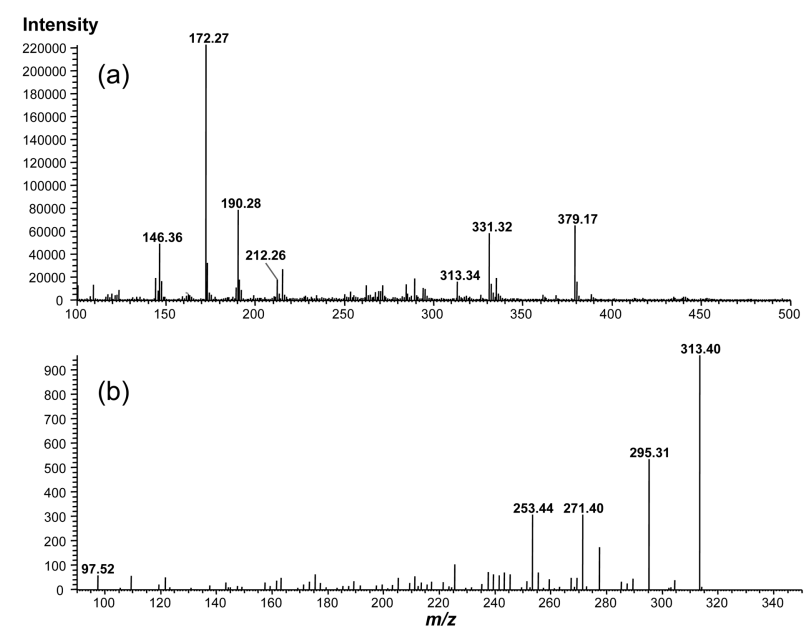

Figure 1. MALDI-MS (a) and MS/MS (b) spectra of $17 \alpha-$ hydroxyprogesterone $(17 \alpha-\mathrm{OHP})$, at the amount of $0.25 \mathrm{ng}$ on the MALDI sample plate, obtained with the CHCA matrix. Distinctive matrix signals for $\alpha$-cyano-4-hydroxycinnamic acid (CHCA) with $17 \alpha-\mathrm{OHP}[\mathrm{M}+\mathrm{H}]^{+}$at $m / z 331$ (a). MALDI-MS/MS of $17 \alpha-\mathrm{OHP}$, the fragmentation of compounds; $\left[\mathrm{M}+\mathrm{H}-\mathrm{H}_{2} \mathrm{O}\right]^{+},\left[\mathrm{M}+\mathrm{H}-2 \mathrm{H}_{2} \mathrm{O}\right]^{+},[\mathrm{M}+\mathrm{H}-$ $60]^{+},[\mathrm{M}+\mathrm{H}-78]^{+},[\mathrm{M}+\mathrm{H}-234]^{+}$at $m / z 313,295,271,253$, and 97 (b). various organic matrices such as dihydroxy benzoic acid (DHB), meso-tetrakis(pentafluorophenyl) porphyrin (F20TPP) and binary matrix in CHCA with DHB and CHCA with 9aminoacridine (9AA) were compared (data not shown), and the analytical efficiency was optimized with $10 \mathrm{mg} / \mathrm{mL}$ of CHCA in $0.2 \%$ trifluoroacetic acid of $70 \%$ acetonitrile.

\section{MALDI-MS analysis of adrenal steroids}

A representative MALDI-LTQ-MS spectrum of $17 \alpha-\mathrm{OHP}$ is shown in Figure 1(a). All adrenal steroids were monitored using their protonated molecular ions, $[\mathrm{M}+\mathrm{H}]^{+}$with characteristic CHCA matrix ions. CHCA own characteristic ions at $m / z 146\left[\mathrm{M}+\mathrm{H}-\mathrm{CN}-\mathrm{H}_{2} \mathrm{O}\right]^{+}, 172\left[\mathrm{M}+\mathrm{H}-\mathrm{H}_{2} \mathrm{O}\right]^{+}, 190[\mathrm{M}+\mathrm{H}]^{+}$, $212[\mathrm{M}+\mathrm{Na}]^{+}$, were $379[2 \mathrm{M}+\mathrm{H}]^{+}$were identified. As an example, $17 \alpha-\mathrm{OHP}$ was detected at $m / z 331[\mathrm{M}+\mathrm{H}]^{+}$and it was selected as the precursor ion for MS/MS analysis in quantitative analysis (Figure 1(b)). Peak identification was achieved by comparing the CHCA matrix peak to avoid overlap with analyte signals. The MS/MS analysis discriminated the target ion from interfering components, especially the CHCA matrix and the existence of the matrix in human serum. Consequently, this MS technique may be conducive to highly reproducible outcomes.

\section{Method validation for MALDI-LTQ-MS/MS analysis of adrenal steroids}

All quantitative results were calculated with the base peak in MS/MS analysis as the quantitative ion (Table 1). The devised method was found to be linear (correlation coefficient, $r 2>0.95$ ) for all analytes, while the LOQ was $12.1 \sim 958.4 \mathrm{ng} / \mathrm{mL}$ in calibration ranges used (Table 2). For full-validation of quantification of adrenal steroids, precision (\% CV) varied from 3.1 to $35.5 \%$, while recoveries ranged from 83.8 to $138.5 \%$ for five different runs in intra-/inter-day (Supplementary Table 1). A quantification of the MALDI technique is controversial for measuring small molecule like steroids; especially as it has high matrix interference to

Table 2. The LOD, LOQ and calibration linearity for 10 adrenal steroids in human serum.

\begin{tabular}{|c|c|c|c|c|}
\hline Compound & $\begin{array}{c}\text { LOD } \\
(\mathrm{ng} / \mathrm{mL})\end{array}$ & $\begin{array}{c}\text { LOQ } \\
(\mathrm{ng} / \mathrm{mL})\end{array}$ & $\begin{array}{c}\text { Calibration } \\
\text { range }(\mathrm{ng} / \mathrm{mL})\end{array}$ & Linearity \\
\hline Progesterone (Prog) & 35.5 & 117.0 & $200-2000$ & 0.991 \\
\hline $17 \alpha-\mathrm{OH}-\mathrm{Prog}$ & 27.3 & 90.1 & $100-2000$ & 0.996 \\
\hline $11 \alpha-\mathrm{OH}-$ Prog & 11.1 & 36.5 & $100-2000$ & 0.995 \\
\hline Cortisol (F) & 290.4 & 958.4 & $500-3000$ & 0.986 \\
\hline Cortisone (E) & 3.6 & 12.1 & $100-1000$ & 0.997 \\
\hline Corticosterone (B) & 45.7 & 150.9 & $200-3000$ & 0.969 \\
\hline 11-DehydroB & 4.3 & 14.1 & $50-1000$ & 0.999 \\
\hline 11-DeoxyB & 27.8 & 91.7 & $100-3000$ & 0.985 \\
\hline 11-DeoxyF & 80 & 263.9 & $100-3000$ & 0.979 \\
\hline Aldosterone & 190.3 & 627.9 & $200-3000$ & 0.952 \\
\hline
\end{tabular}

Mass Spectrom. Lett. 2011 Vol. 2, No. 3, 69-72 


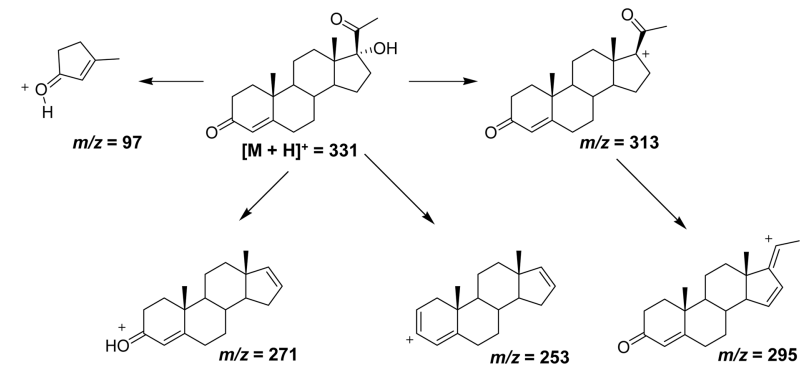

Figure 2. The MS/MS fragmentation of $17 \alpha$-hydroxyprogesterone.

the analyte and low reproducibility between the target samples. However, successful quantification of adrenal steroids from serum samples by MLADI-LTQ-MS/MS was achieved via isotope-labeled internal standards to improve experimental reproducibility. $^{14}$

The storage stability of adrenal steroids was measured by freeze-thawing for 42 and 72 hours respectively; or by shortterm storage in $6 \mathrm{hr}$ at room temperature. Even after 42 and $72 \mathrm{~h}$ for freeze and thaw cycle condition, the concentrations of all analytes at room temperature had shown reproducible data, and also in short term storage in $6 \mathrm{hr}$ at room temperature in MALDI-MS/MS analysis (Supplementary Table 2).

\section{Application to congenital adrenal hyperplasia}

The devised method was applied to quantify 10 adrenal steroids in serum obtained from $\mathrm{CAH}$ patients. Since the target $17 \alpha-\mathrm{OHP}$ concentration range suggested for $\mathrm{CAH}$ is 20 $2000 \mathrm{ng} / \mathrm{mL}$, in general, average concentration of $17 \alpha-\mathrm{OHP}$ level is more than $100 \mathrm{ng} / \mathrm{mL}$ of the concentration. ${ }^{13,15,16}$ Therefore, the present MALDI-MS/MS method could produce quantification of $17 \alpha-\mathrm{OHP}$ for $\mathrm{CAH}$ detection. Accordingly, MALDI-MS/MS can be use as a measurement of $17 \alpha-O H P$ for a high-throughput and sensitive $\mathrm{CAH}$ diagnostic tool.

\section{Conclusions}

A high throughput screening method for adrenal steroid analysis by MALDI-MS/MS was developed and validated. For adrenal steroids, this analytical method could improve reproducibility, which is one disadvantage of MALDI analysis.
Therefore, this method is a very promising tool for rapid quantitative analysis for $17 \alpha-\mathrm{OHP}$ quantification in $\mathrm{CAH}$ patients. The high speed of analysis and overall sensitivity of MALDI-MS makes it a viable alternative technique to the other traditional methods in steroid analysis.

\section{Acknowledgements}

This study was supported by an intramural grant from Korea Institute of Science and Technology (KIST) and by the Converging Research Center Program through the Ministry of Education, Science and Technology (2011K000885).

\section{References}

1. Riepe, F. G.; Sippell, W. G. Rev. Endocr. Metab Disord. 2007, 8, 349.

2. Wang, Y.; Hornshaw, M.; Alvelius, G.; Bodin, K.; Liu, S.; Sjovall, J.; Griffiths, W. J. Anal. Chem. 2006, 78, 164.

3. Ha, Y. W.; Moon, J. M.; Jung, H. J.; Chung, B. C.; Choi, M. H. J. Chromatogr. B 2009, 877, 4125.

4. El-Aneed, A.; Cohen, A.; Banoub, J. Appl. Spectro. Rev. 2009, 44, 210.

5. Galesio, M.; Rial-Otero, R.; Capelo-Martinez, J. -L. Rapid. Commun. Mass Spectrom. 2009, 23, 1783.

6. Sleno, L; Bolmer, D. A. Rapid. Commun. Mass Spectrom. 2005, 19, 1928.

7. Tholey, A.; Wittmann, C.; Kang, M.; Bungert, D.; Hollemeyer, K.; Heinzle, E. J. Mass Spectrom. 2002, 37, 963.

8. Kosanam, K.; Sai Prakash, P. K.; Yates, C. R.; Miller, D. D.; Ramagiri, S. Anal. Chem. 2007, 79, 6020.

9. Kampen, J. J.; Luider, A. T. M.; Ruttink, P. J. A.; Burgers, P. C. J. Mass Spectrom. 2009, 44, 1556.

10. Guo, Z,; He, L. Anal. Bioanal. Chem. 2007, 387, 1939.

11. Lastovickova, M.; Chmelik, J.; Bovalova, J. Int. J. Mass Spectrom. 2009, 281, 82.

12. Carlson, A. D.; Obeid, J. S.; Kanellopoulou, N.; Wilson, R. C.; New, M. I. J. Steroid Biochem. Mol. Biol. 1999, 69, 19.

13. Merke, D. P.; Bornstein, S. R. Lancet 2005, 365, 2125.

14. Gobey, J.; Cole, M.; Janiszewski, J.; Covey, T.; Chau, T.; Kovarik, P.; Corr, J. Anal. Chem. 2005, 77, 5643.

15. White, P. C.; Speiser, P. W. Endocr. Rev. 2000, 21, 245.

16. Forest, M. G. Hum. Reprod. Update 2004, 10, 469. 